Article

\title{
Asymmetric Split-Gate 4H-SiC MOSFET with Embedded Schottky Barrier Diode for High-Frequency Applications
}

\author{
Kyuhyun Cha and Kwangsoo Kim *(D) \\ Department of Electronic Engineering, Sogang University, Seoul 04107, Korea; bksem10ckh25@naver.com \\ * Correspondence: kimks@sogang.ac.kr; Tel.: +82-27-05-8913
}

check for updates

Citation: Cha, K.; Kim, K.

Asymmetric Split-Gate 4H-SiC MOSFET with Embedded Schottky Barrier Diode for High-Frequency Applications. Energies 2021, 14, 7305. https://doi.org/10.3390/en14217305

Academic Editors: Ka Wai Eric Cheng and Bertrand Lenoir

Received: 5 October 2021

Accepted: 2 November 2021

Published: 4 November 2021

Publisher's Note: MDPI stays neutral with regard to jurisdictional claims in published maps and institutional affiliations.

Copyright: (c) 2021 by the authors. Licensee MDPI, Basel, Switzerland. This article is an open access article distributed under the terms and conditions of the Creative Commons Attribution (CC BY) license (https:/ / creativecommons.org/licenses/by/ $4.0 /)$.

\begin{abstract}
H-SiC Metal-Oxide-Semiconductor Field Effect Transistors (MOSFETs) with embedded Schottky barrier diodes are widely known to improve switching energy loss by reducing reverse recovery characteristics. However, it weakens the static characteristics such as specific on-resistance and breakdown voltage. To solve this problem, in this paper, an Asymmetric 4H-SiC Split Gate MOSFET with embedded Schottky barrier diode (ASG-MOSFET) is proposed and analyzed by conducting a numerical TCAD simulation. Due to the asymmetric structure of ASG-MOSFET, it has a relatively narrow junction field-effect transistor width. Therefore, despite using the split gate structure, it effectively protects the gate oxide by dispersing the high drain voltage. The Schottky barrier diode (SBD) is also embedded next to the gate and above the Junction Field Effect transistor (JFET) region. Accordingly, since the SBD and the MOSFET share a current path, the embedded SBD does not increase in $R_{O N, S P}$ of MOSFET. Therefore, ASG-MOSFET improves both static and switching characteristics at the same time. As a result, compared to the conventional 4H-SiC MOSFET with embedded SBD, Baliga's Figure of Merit is improved by 17\%, and the total energy loss is reduced by $30.5 \%$, respectively.
\end{abstract}

Keywords: 4H-SiC; asymmetric; split gate; body diode; switching loss

\section{Introduction}

$4 \mathrm{H}-\mathrm{SiC}$ is a wide bandgap material and has material properties such as high critical electric field and thermal conductivity [1]. With these characteristics, the use of $4 \mathrm{H}-\mathrm{SiC}$ enables the implementation of MOSFETs, which significantly improves the switching characteristics compared to the conventional Si IGBT [2,3]. Therefore, 4H-SiC MetalOxide-Semiconductor Field Effect Transistors (MOSFETs) are considered to be promising candidates for high-voltage and high-frequency applications. In particular, high-voltage and high-frequency power devices are expected to be applied in applications requiring high-power density, such as high-power converters and traction drives [4,5]. However, most commercially available SiC power MOSFETs are for $1200 \mathrm{~V}$ and $1700 \mathrm{~V}$ applications. In response to these industry demands, SiC MOSFETs for $3300 \mathrm{~V}$ are being actively researched [6,7]. Recently, various studies into trench MOSFETs have been conducted due to the high channel mobility and the small cell pitch of trench MOSFETs. However, trench MOSFETs suffer from electric field concentrations at the trench gate oxide corners exceeding $3 \mathrm{MV} / \mathrm{cm}$, the gate oxide reliability limit of SiC MOSFETs [8]. Therefore, for high voltage applications, research mainly focuses on planar MOSFETs.

To achieve fast switching speeds and low switching energy losses for high frequency, the gate-drain capacitance $\left(C_{G D}\right)$ must be minimized [9]. A well-known method to reduce $C_{G D}$ of the planar MOSFET is a split gate MOSFET, which reduces the overlapped region between gate and drain through the split active gate [10]. However, in this structure, the electric field is concentrated at the corner of the split gate oxide, so there is a problem in that the reliability of the gate oxide cannot be guaranteed. In particular, this problem becomes more serious as the high-voltage device increases. Therefore, the split gate structure is difficult to apply to high voltage applications $(>3.3 \mathrm{kV})$. 
Another way to improve the switching characteristic is to improve the reverse recovery characteristics. Because the body diode of SiC MOSFETs has a high reverse recovery charge, a high reverse recovery current is generated during the turn on transient in the switching operation [11]. The reverse recovery current increases the peak drain current, resulting in high switching energy loss. To handle the reverse recovery current, an external Schottky barrier diode (SBD) is widely used as a freewheeling diode to suppress the action of the body diode [12]. However, an external SBD increases the active chip area and package cost. In high voltage applications, the active chip area of external SBD increases as the size of the external SBD increases to withstand the high voltage in the off state [13]. Recently, to improve the reverse recovery characteristics of SiC MOSFETs without using an external SBD, research into embedding SBD in SiC MOSFET has been actively conducted. A conventional 4H-SiC MOSFET with embedded SBD (C-MOSFET) greatly improves reverse recovery characteristics without external SBD [14]. However, embedded SBD increases the cell pitch, decreasing the channel density and thus increases the specific onresistance $\left(R_{O N, S P}\right)$. Moreover, in the off state, due to the increased mesa region, the voltage concentrated on the $\mathrm{p}+$ base is also increased, reducing the Breakdown Voltage $(B V)$. That is, the SBD embedded in the mesa region improves the reverse recovery characteristic but is accompanied by a deterioration of the static characteristics.

In this paper, we propose an asymmetric split gate $4 \mathrm{H}-\mathrm{SiC}$ MOSFET with embedded SBD (ASG-MOSFET) to simultaneously improve both the switching and static characteristics. In ASG-MOSFET, the SBD is embedded next to the active gate and above the junction field effect transistor (JFET) region. Accordingly, the embedded SBD does not degrade the $R_{O N, S P}$ of MOSFET because the MOSFET and SBD share the current path in the on state. In addition, since the ASG-MOSFET is an asymmetric structure, it has a narrow JFET width $\left(W_{\text {JFET }}\right)$. Therefore, it has a high channel density through a small cell pitch, thus reducing the $R_{O N, S P}$ despite the narrow $W_{J F E T}$. This narrow $W_{J F E T}$ also forms a large depletion region, creating a small depletion capacitance $\left(C_{D E P}\right)$. Moreover, the split gate structure of ASG-MOSFET reduces the overlapping area between gate and drain, thereby reducing gate oxide capacitance $\left(C_{O X}\right)$. Therefore, ASG-MOSFET significantly reduces $C_{G D}$ through the reduced $C_{O X}$ and $C_{D E P}$.

This study was conducted by a Sentaurus TCAD simulation tool. In this simulation, the used models include the Fermi-Dirac statistics, band narrowing, anisotropic material properties, Shockley-Read-Hall, Auger recombination. A Hatakeyama model is used to consider the impact of ionization behavior. Mobility models, such as doping dependent, high field saturation, surface roughness, and acoustic phonon scattering at the $\mathrm{SiC} / \mathrm{SiO}_{2}$ interface are involved [15]. The incomplete ionization model is also considered and the nonlocal tunneling model and Schottky barrier lowering model are used for the tunneling current at the Schottky contact [16]. The fixed charge concentration at the $\mathrm{SiC} / \mathrm{SiO}_{2}$ interface is considered as $3 \times 10^{12} \mathrm{~cm}^{-2}$.

\section{Device Structure and Optimization}

\subsection{Proposed Device Structure}

Figure 1 is a cross-sectional view of the C-MOSFET and the proposed ASG-MOSFET. In Figure 1, the cells used for the simulation are marked with a red dotted line. In Figure 1, the C-MOSFET and the ASG-MOSFET have the SBD embedded over the mesa region and the JFET region, respectively. 


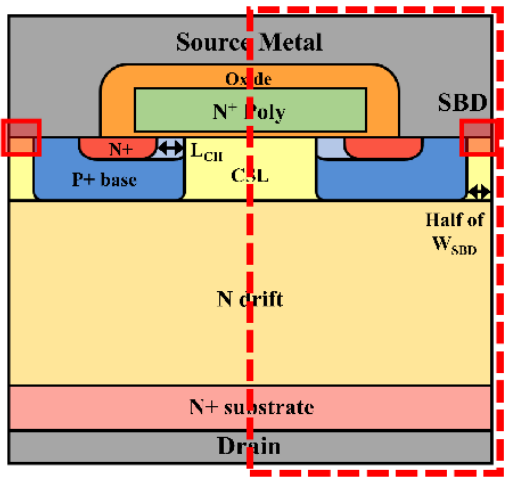

(a)

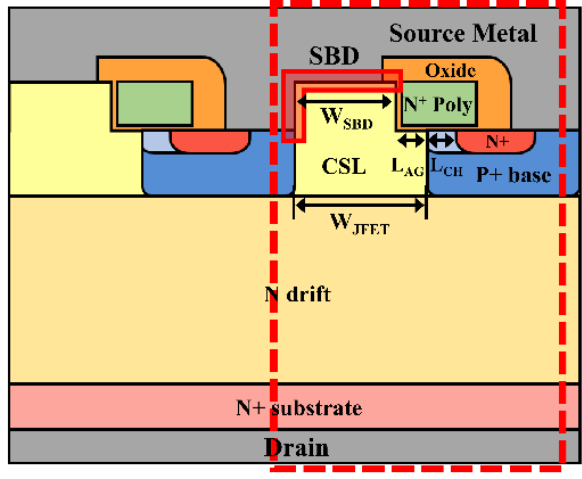

(b)

Figure 1. Schematic cross-sectional views (a) conventional MOSFET with embedded SBD (CMOSFET) and (b) asymmetric split gate MOSFET with embedded SBD (ASG-MOSFET).

For the two device structures, the channel length and gate oxide thicknesses are $0.5 \mu \mathrm{m}$ and $50 \mathrm{~nm}$, respectively, and the doping concentration in the channel region is $2 \times 10^{17} \mathrm{~cm}^{-3}$. Considering the simultaneous formation of ohmic and Schottky contacts using $\mathrm{Ni}$ as a source metal, the work function of the Schottky metal was set to $5.05 \mathrm{eV}$ [17]. The electron mobility is set at about $20-50 \mathrm{~cm}^{2} \mathrm{~V}^{-1} \mathrm{~S}^{-1}$ in the $15 \mathrm{~nm}$ range below the $\mathrm{SiC} / \mathrm{SiO}_{2}$ interface. A P+ base region doping concentration of $2 \times 10^{18} \mathrm{~cm}^{-3}$, an N-drift layer of $30 \mu \mathrm{m}$, an N-drift doping concentration of $3 \times 10^{15} \mathrm{~cm}^{-3}$, and a Channel Spread Layer (CSL) doping concentration of $2 \times 10^{16} \mathrm{~cm}^{-3}$ were used. A heavily doped $1 \times 10^{20}$ $\mathrm{cm}^{-3} \mathrm{~N}$-type Poly Si was adopted as the active gate. The $W_{\text {JFET }}$ of C-MOSFET was set to $2.0 \mu \mathrm{m}$ in consideration of static characteristics such as $R_{O N, S P}$, and $B V$. The gate oxide thickness of the two structures was considered to be $50 \mathrm{~nm}$.

\subsection{Key Parameter Optimization}

In this section, optimization of the two structures was performed considering $R_{O N, S P}$ and turn on voltage of embedded SBD $\left(V_{F, S B D}\right)$ of C-MOSFET and ASG-MOSFET. As previously mentioned, the C-MOSFET improves the reverse recovery characteristic through the embedded SBD, but it accompanies the degradation of the static characteristic. Therefore, the SBD width $\left(W_{S B D}\right)$ of C-MOSFET was designed considering $R_{O N, S P}$ and $V_{F, S B D}$. Figure 2 shows $R_{O N, S P}$ and $V_{F, S B D}$ according to $W_{S B D}$ change in C-MOSFET. As $W_{S B D}$ increases, $V_{F, S B D}$ decreases while $R_{O N, S P}$ increases. This is because the embedded SBD in the mesa region increases the cell pitch of the C-MOSFET, reducing the channel density. Therefore, considering both $R_{O N, S P}$ and $V_{F, S B D}, 2.0 \mu \mathrm{m}$ with the most appropriate performance was determined as the $W_{S B D}$.

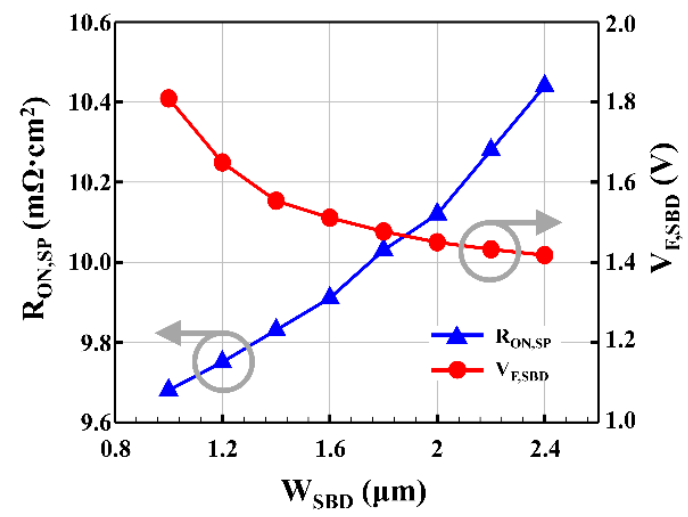

Figure 2. Trade-off between $R_{O N, S P}$ and $V_{F, S B D}$ of C-MOSFET according to $W_{S B D}$. 
For a fair comparison between C-MOSFET and ASG-MOSFET, the $W_{J F E T}$ of ASGMOSFET is designed such that the BV of the ASG-MOSFET has a BV similar to that of the C-MOSFET. In the ASG-MOSFET, the $W_{J F E T}$ consists of $W_{S B D}$, the active gate length $\left(L_{A G}\right)$ and the gate oxide thickness $\left(T_{O X}\right)$. The $L_{A G}$ and $T_{O X}$ of ASG-MOSFET is fixed to $0.2 \mu \mathrm{m}$ and 50nm. Therefore, the $W_{J F E T}$ of the ASG-MOSFET was optimized by adjusting the $W_{S B D}$.

Figure 3 shows the effect of $W_{J F E T}$ on static characteristics of the ASG-MOSFET. In Figure 3a, unlike C-MOSFET, when $W_{S B D}$ increases, $V_{F, S B D}$ and $R_{O N, S P}$ decrease simultaneously. In other words, the embedded SBD of the ASG-MOSFET does not degrade the $R_{O N, S P}$ characteristic of the ASG-MOSFET. This is because the MOSFET channel and the embedded SBD share a current path through the JFET region in their respective on state. Meanwhile, from Figure $3 b$, in the off state, as the $W_{J F E T}$ increases, the $B V$ decreases due to the reduction of the screening effect by the JFET region. As a result, $1.4 \mu \mathrm{m}$, the condition with the most similar $B V$ to that of C-MOSFET, was determined as $W_{J F E T}$. Therefore, $W_{S B D}$, $L_{A G}$, and $T_{O X}$ of the optimized ASG-MOSFET were designed to be $1.4 \mu \mathrm{m}, 0.2 \mu \mathrm{m}$, and $50 \mathrm{~nm}$, respectively.

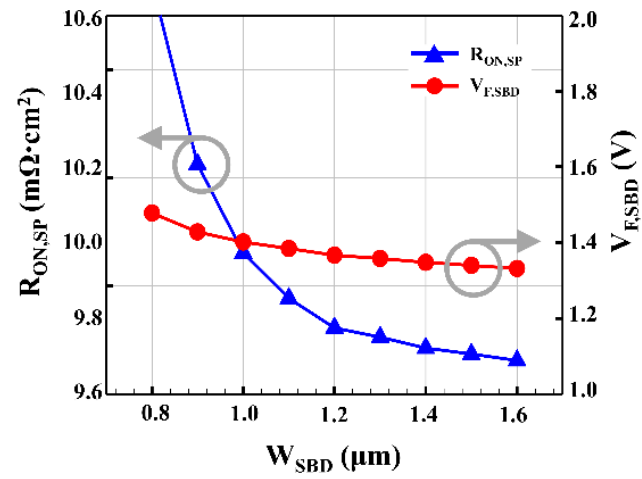

(a)

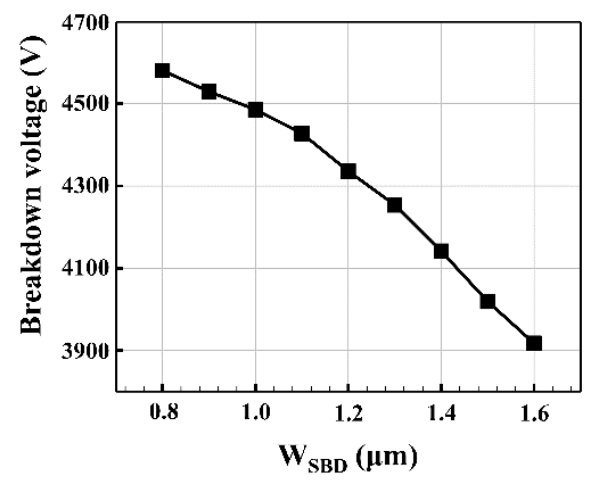

(b)

Figure 3. Static characteristics of ASG-MOSFET according to $W_{S B D}$ (a) $R_{O N, S P}$ and $V_{F, S B D}$ characteristics and (b) $B V$.

\section{Electrical Characteristics Analysis}

\subsection{Static Characteristics}

In this section, to verify the excellent electrical properties of the proposed ASGMOSFET, the electrical properties of the two structures were compared and analyzed through TCAD simulation.

Figure 4 shows output curves and $B V$ characteristics of the C-MOSFET and ASGMOSFET. According to the results optimized in Section 2.2, C-MOSFET and ASG-MOSFET have similar BV of $4094 \mathrm{~V}$ and $4141 \mathrm{~V}$, respectively. Whereas, The $R_{O N, S P}$ of both structures are $10.12 \mathrm{~m} \Omega \cdot \mathrm{cm}^{2}$ and $8.85 \mathrm{~m} \Omega \cdot \mathrm{cm}^{2}$, respectively. ASG-MOSFET has higher JFET resistance than C-MOSFET due to smaller $W_{J F E T}$, but $R_{O N, S P}$ of ASG-MOSFET is $12.5 \%$ smaller than C-MOSFET. This is because the ASG-MOSFET has a smaller cell pitch, which increases the channel density. As a result, the BFOM calculated by $B V^{2} / R_{O N, S P}$ improved by $17 \%$, respectively, compared to C-MOSFET.

The electric field distributions of C-MOSFET and ASG-MOSFET when $V_{D S}=3000 \mathrm{~V}$ are plotted in Figure 5a,b, respectively. The C-MOSFET has the highest gate oxide electric field $\left(E_{O X}\right)$ in the center of the gate oxide because the $W_{J F E T}$ is wide. On the other hand, in ASG-MOSFET, the split gate corner is most vulnerable to electric field. However, because the ASG-MOSFET has an asymmetric structure, the gate oxide is more effectively protected by forming a wide depletion region in the JFET region through a narrow $W_{J F E T}$. As a result, the maximum $E_{O X}$ of C-MOSFET and ASG-MOSFET is $2.74 \mathrm{MV} / \mathrm{cm}$ and $2.02 \mathrm{MV} / \mathrm{cm}$, respectively, so ASG-MOSFET has a more superior gate oxide reliability. 


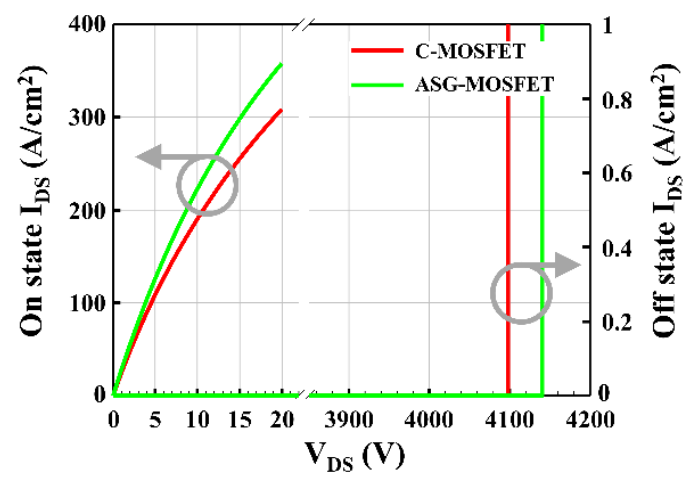

Figure 4. Output curve and $B V$ characteristics of C-MOSFET and ASG-MOSFET.

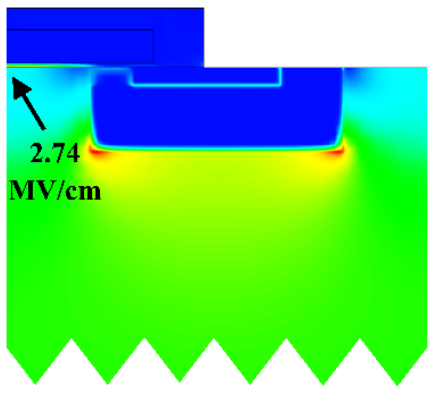

(a)

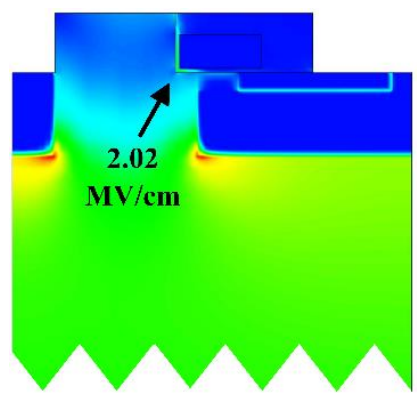

(b)

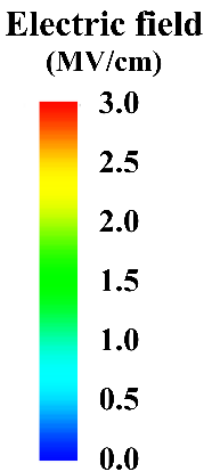

0.0

Figure 5. Electric field distribution of (a) C-MOSFET and (b) ASG-MOSFET when $V_{D S}=3000 \mathrm{~V}$.

Figure 6 shows the forward conduction characteristics of the body diode. The forward conduction characteristics were extracted by sweeping $V_{D S}$ from $0 \mathrm{~V}$ to $-10 \mathrm{~V}$ when $V_{G S}=-5 \mathrm{~V}$. In Figure 6, the $V_{F, S B D}$ is defined as the $V_{D S}$ when the drain current reaches $-80 \mathrm{~A} / \mathrm{cm}^{2}$. Meanwhile, the knee point means the point at which the body diode is turned on [18]. As a result, $V_{F, S B D}$ of C-MOSFET is $1.45 \mathrm{~V}$, whereas $V_{F, S B D}$ of ASG-MOSFET is $1.35 \mathrm{~V}$. Therefore, the ASG-MOSFET more effectively suppresses the turn on of the body diode. The static characteristics of the two devices, including $R_{O N, S P}, B V$, maximum $E_{O X}$, $V_{F, S B D}$ and turn on voltage of body diode $\left(V_{F, B o d y}\right)$, are summarized in Table 1 . In addition, Baliga's Figure of Merit (BFOM), which is the static characteristic indicator considering $R_{O N, S P}$ and $B V$, was calculated and listed.

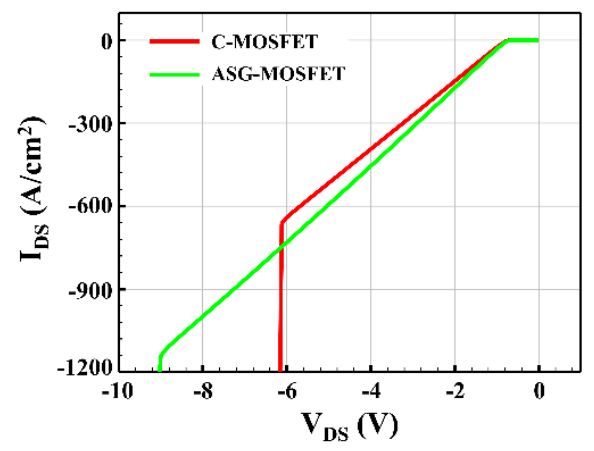

Figure 6. Forward conduction of the body diode of C-MOSFET and ASG-MOSFET. 
Table 1. Dynamic characteristics of two devices.

\begin{tabular}{ccc}
\hline & C-MOSFET & ASG-MOSFET \\
\hline$R_{O N, S P}\left(\mathrm{~m} \Omega \cdot \mathrm{cm}^{2}\right)$ & 10.12 & 8.85 \\
$B V(\mathrm{~V})$ & 4098 & 4141 \\
$B F O M\left(\mathrm{MW} / \mathrm{cm}^{2}\right)$ & 1659.5 & 1937.6 \\
Maximum $E_{O X}(\mathrm{MV} / \mathrm{cm})$ & 2.74 & 2.02 \\
$V_{F, S B D}(\mathrm{~V})$ & 1.45 & 1.35 \\
$V_{F, B \text { ody }}(\mathrm{V})$ & 6.12 & 9.0 \\
\hline
\end{tabular}

\subsection{Dynamic Characteristics}

The capacitance simulation results of two devices are depicted in Figure 7. In the capacitance simulation, the capacitance was extracted by sweeping the drain voltage from $0 \mathrm{~V}$ to $1500 \mathrm{~V}$ when $V_{G S}=0 \mathrm{~V}$, and the AC small signal was set to $1 \mathrm{MHz}$. In addition, the active area of two devices of two devices is set to $1 \mathrm{~cm}^{2}$.

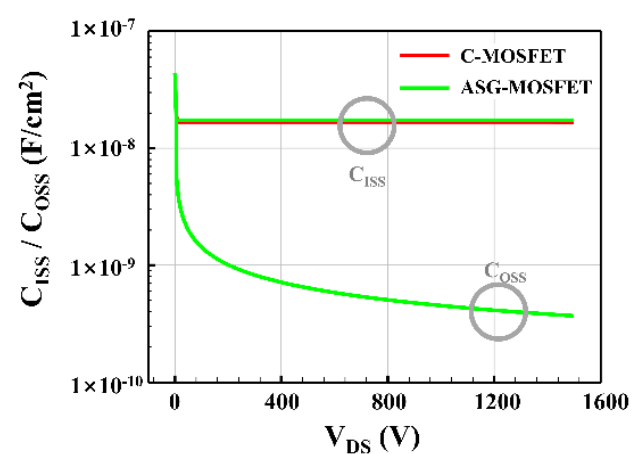

(a)

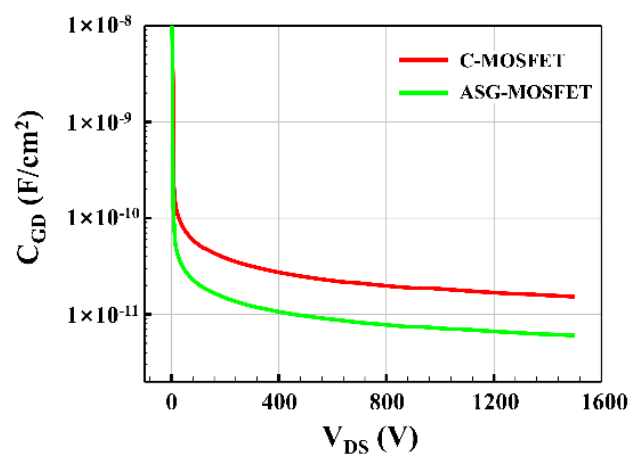

(b)

Figure 7. Capacitance of C-MOSFET and ASG-MOSFET (a) Input and output capacitance and (b) Gate-drain capacitance.

From Figure $7 \mathrm{a}$, it can be seen that the input capacitance $\left(C_{I S S}\right)$ and the output capacitance $\left(C_{O S S}\right)$ of two structures are very similar. In the Figure $7 \mathrm{~b}$, the ASG-MOSFET has a significant improvement in $C_{G D}$ over C-MSOFET. The $C_{G D}$ of ASG-MOSFET is $6.04 \mathrm{pF} / \mathrm{cm}^{2}$, which is improved by $60.4 \%$ compared to $15.24 \mathrm{pF} / \mathrm{cm}^{2}$ of C-MOSFET. This is because the ASG-MOSFET greatly reduces the $C_{O X}$ and $C_{D E P}$ constituting $C_{G D}$. The $C_{G D}$ is a series connection of $C_{O X}$ and $C_{D E P}$ [9]. The split-gate structure of ASG-MOSFET effectively reduces the overlapping area between gate and drain, reducing the $C_{O X}$. In addition, narrow $W_{\text {JFET }}$ of ASG-MOSFET forms a wide depletion region between gate and drain, which also reduces $C_{D E P}$. Therefore, the ASG-MOSFET has significantly reduced $C_{G D}$. Based on the $R_{O N, S P}$ and $C_{G D}$ results, High-Frequency Figure of Merit (HFFOM), which is commonly used as the high frequency performance indicators is calculated [2]. As a result, owing to the reduced $R_{O N, S P}$ and $C_{G D}$, the HFFOM of the ASG-MOSFET is $53.45 \mathrm{~m} \Omega \cdot \mathrm{pF}$, which is a $65 \%$ improvement compared to the $154.23 \mathrm{~m} \Omega \cdot \mathrm{pF}$ of the C-MOSFET.

Figure 8 shows the switching waveforms of both devices when used as a device under test (DUT) in a half-bridge circuit. Figure 8a shows this during the turn on transient and turn off transient, respectively, and Figure $8 \mathrm{~b}$ appears to show the half bridge circuits used for the double pulse test simulation. In Figure $8 b$, the gate resistance is set to $10 \Omega$ and the gate voltage switched from $-5 \mathrm{~V}$ to $20 \mathrm{~V}$. In order to proceed with the double pulse test of the $3.3 \mathrm{kV}$ device, $\mathrm{V}_{\mathrm{DD}}$ was set to $1700 \mathrm{~V}$. Furthermore, to set the load current to $100 \mathrm{~A} / \mathrm{cm}^{2}$ in the test circuit, the time period of the first pulse and load inductance are set to $10 \mu$ s and $170 \mu \mathrm{H}$, respectively, considering the $\mathrm{V}_{\mathrm{DD}}$ and the rate of di/dt, and a parasitic inductance is assumed to $10 \mathrm{nH}$. In addition, the same MOSFET as the lower arm MOSFET was used for the upper arm MOSFET to handle the freewheeling current in the half-bridge circuit, and $V_{G S}$ of the upper arm MOSFET was applied to $-5 \mathrm{~V}$ to maintain the off state of the 
upper arm MOSFET. The SBD connected in parallel to the MOSFET in a half-bridge circuit appears in the SBD, which is embedded into the MOSFET.

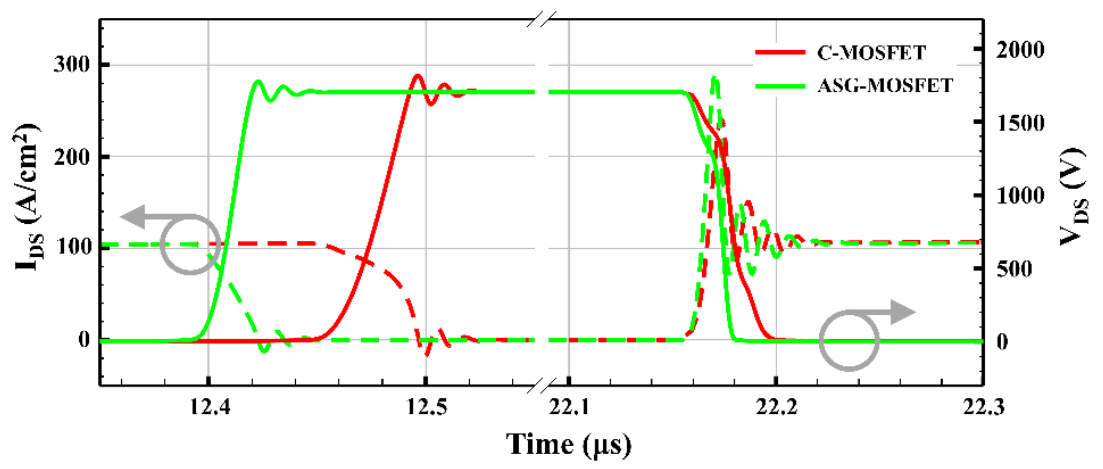

(a)

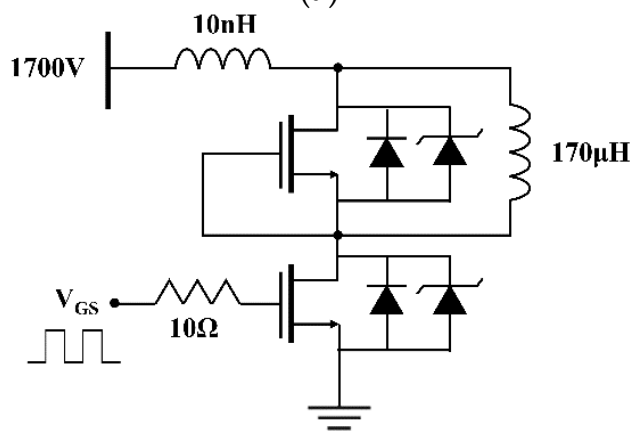

(b)

Figure 8. (a) Switching waveforms of C-MOSFET and ASG-MOSFET during turn off transient and turn on transient and (b) half-bridge circuit used for double pulse test simulation.

In Figure 8a, the ASG-MOSFET has a shorter Miller plateau and a faster switching speed than C-MOSFET during the switching transients, which is consistent with the $C_{G D}$ results. Meanwhile, although SBD of ASG-MOSFET has lower $V_{F, S B D}$ than C-MOSFET, the peak current during switching transient is larger. This is because the fast-switching time of the ASG-MOSFET results in a higher overshoot current [19].

Figure 9 shows the current waveform of upper arm MOSFET during the turn on transient of lower arm MOSFET when the C-MOSFET and ASG-MOSFET are used as DUT in half-bridge circuit. From Figure 9, the ASG-MOSFET has a slightly larger reverse recovery charge $\left(Q_{R R}\right)$ compared to the C-MOSFET. This is because the reduced $C_{G D}$ of the ASG-MOSFET causes a larger overshoot current, resulting in a higher peak current.

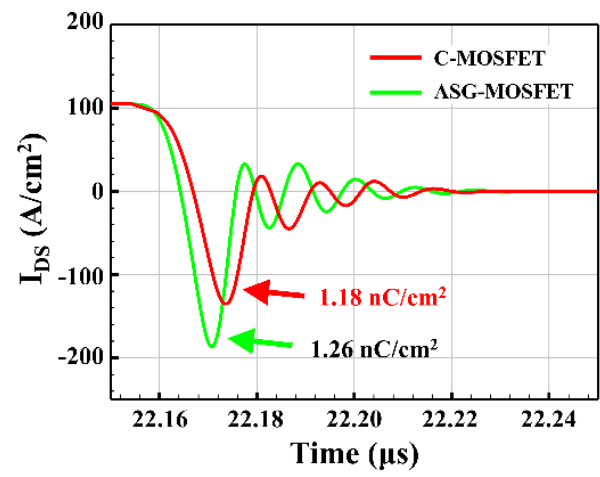

Figure 9. Current waveform of upper arm MOSFET during the turn on transient of lower arm DUT.

The ASG-MOSFET has a slightly larger $Q_{R R}$, but more superior the switching characteristics than the C-MOSFET. Figure 10 shows the total switching energy loss $\left(E_{\text {Total }}\right)$ of two 
devices during the switching transient. The $E_{\text {Total }}$ of the ASG-MOSFET is still lower than that of the C-MOSFET shown in Figure 10 due to its fast-switching speed. Based on the double pulse test simulation results, the turn off loss $\left(E_{O F F}\right)$ and the turn on loss $\left(E_{O N}\right)$ of ASG-MOSFET are $0.84 \mathrm{~mJ} / \mathrm{cm}^{2}$ and $3.19 \mathrm{~mJ} / \mathrm{cm}^{2}$, respectively, whereas the C-MOSFET has an $E_{\text {OFF }}$ of $1.79 \mathrm{~mJ} / \mathrm{cm}^{2}$ and an $E_{O N}$ of $4.01 \mathrm{~mJ} / \mathrm{cm}^{2}$, the $E_{\text {Total }}$ of ASG-MOSFET is $4.03 \mathrm{~mJ}$, which is $30.5 \%$ lower than that of C-MOSFET with $E_{\text {Total }}$ of $5.80 \mathrm{~mJ}$. As a result, the switching characteristics, including the parasitic capacitance and reverse recovery characteristics of the two devices, are summarized in Table 2.

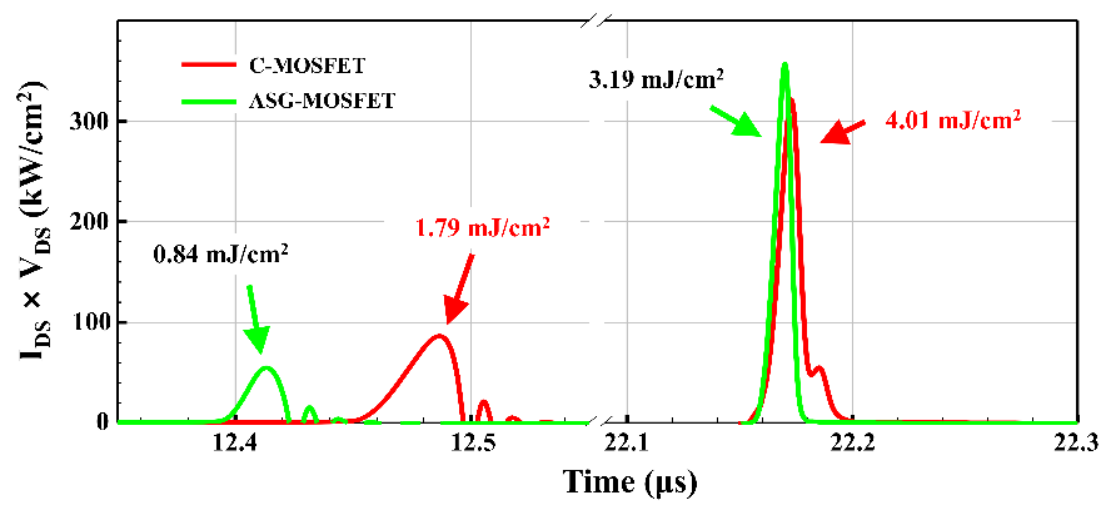

Figure 10. Switching energy loss during turn off and turn on transients.

Table 2. Dynamic characteristics of two devices.

\begin{tabular}{ccc}
\hline & C-MOSFET & ASG-MOSFET \\
\hline$C_{I S S}\left(\mathrm{nF} / \mathrm{cm}^{2}\right)$ & 16.79 & 17.34 \\
$C_{O S S}\left(\mathrm{pF} / \mathrm{cm}^{2}\right)$ & 369.79 & 369.71 \\
$C_{G D}\left(\mathrm{pF} / \mathrm{cm}^{2}\right)$ & 15.24 & 6.04 \\
$Q_{R R}\left(\mathrm{nC} / \mathrm{cm}^{2}\right)$ & 1.18 & 1.26 \\
$E_{O F F}\left(\mathrm{~mJ} / \mathrm{cm}^{2}\right)$ & 1.79 & 0.84 \\
$E_{\text {ON }}\left(\mathrm{mJ} / \mathrm{cm}^{2}\right)$ & 4.01 & 3.19 \\
$E_{\text {Total }}\left(\mathrm{mJ} / \mathrm{cm}^{2}\right)$ & 5.80 & 4.03 \\
\hline
\end{tabular}

\section{Proposed Fabrication Process}

Considering the feasibility of the proposed MOSFE, the fabrication process of ASGMOSFET is proposed as shown in Figure 11. The N- drift and Channel Spread Layer are grown on an $\mathrm{n}$ - substrate by epitaxial process, and the CSL layer is etched to remove the CSL layer, except for the Schottky contact region. N+ source, P+ base and P channel region are formed by the implantation process. The chemical vapor deposition is performed at $800{ }^{\circ} \mathrm{C}$ to form an oxide with a uniform thickness of $50 \mathrm{~nm}$ [20]. Poly Si is deposited by the low-pressure chemical vapor deposition (LPCVD) and is etched by reactive ion etching to form a split gate structure [21]. Interlayer dielectric oxide is deposited through LPCVD and is etched to open the Ohmic and Schottky contact region [22]. After Ni is deposited, rapid thermal annealing (RTA) is performed at $900{ }^{\circ} \mathrm{C}$ [23]. RTA at $900{ }^{\circ} \mathrm{C}$ after $\mathrm{Ni}$ deposition allows $\mathrm{Ni}$ to serve as a multifunctional metal that simultaneously forms Ohmic and Schottky contacts. Finally, an Ni layer is patterned, and a thick Al layer is deposited to form a metal pad. 


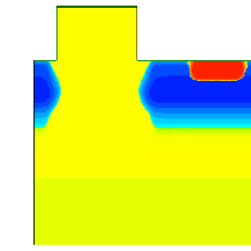

(a) $\mathrm{SiC}$ epitaxial and etching \& Ion implantation

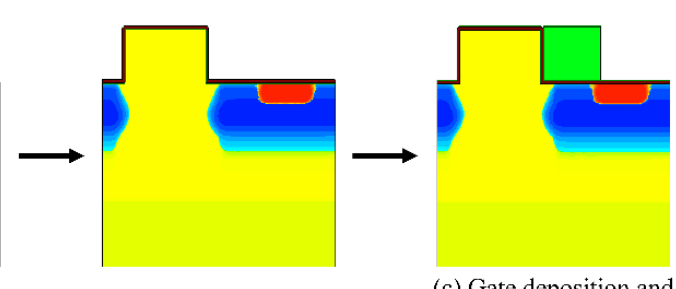

(b) Gate oxide deposition (c) Gate deposition and patterning

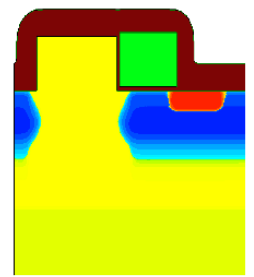

(d) ILD oxide deposition

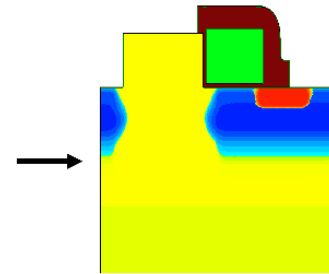

(e) ILD oxide patterning

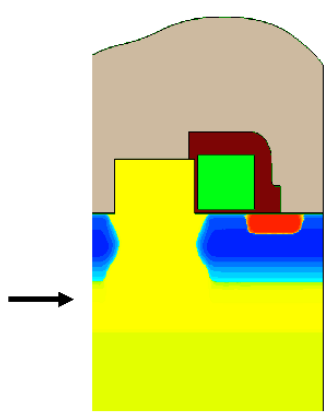

(f) Metallization

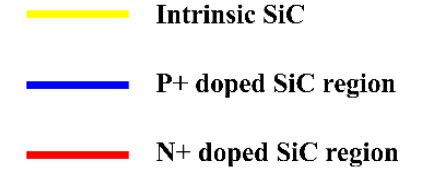

Oxide

Gate Poly Si

Source Metal

Figure 11. Proposed process flow of ASG-MOSFET. (a) Epitaxial N-drift layer and CSL on N+ substrate and Form the P+ base, $\mathrm{P}$ channel and $\mathrm{N}+$ source by implantation (b) gate oxide deposition (c) Poly Si deposition and patterning (d) ILD deposition (e) ILD patterning (f) Ni metal deposition to form the ohmic and Schottky contact.

\section{Conclusions}

In this paper, based on the TCAD simulation results, we propose and analyze an asymmetric $4 \mathrm{H}-\mathrm{SiC}$ split-gate MOSFET with embedded SBD (ASG-MOSFET). ASG-MOSFET has narrower $W_{\text {JFET }}$ than C-MOSFET due to its asymmetric structure. The narrower $W_{\text {JFET }}$ forms a wide depletion region, causing a better shielding effect of the JFET region. Therefore, the split gate structure of ASG-MOSFET is effectively protected without degradation of $\mathrm{BV}$ and gate oxide reliability. ASG-MOSFET also has lower $R_{O N, S P}$ than C-MOSFET. Unlike C-MOSFET, in ASG-MOSFET, the SBD is embedded above the JFET region so that the embedded SBD does not degrade the $R_{O N, S P}$ and the ASG-MOSFET has a smaller cell pitch, increasing channel density. In addition, since narrow $W_{\text {JFET }}$ due to asymmetric structure effectively reduces $C_{D E P}$, ASG-MOSFET effectively improves switching characteristics through split gate, narrow $W_{\text {JFET }}$ due to its asymmetric structure, and embedded SBD. As a result, ASG-MOSFET improved BFOM and switching energy loss by $17 \%$ and $30.5 \%$, respectively, compared to C-MOSFET. Therefore, ASG-MOSFET is more suitable for high voltage and high frequency applications.

Author Contributions: All authors contributed to this work. Please confirm. Conceptualization, K.C.; methodology, K.C. and K.K.; software, K.C.; validation, K.C. and K.K.; formal analysis, K.C. and K.K.; investigation, K.C.; resources, K.C. and K.K.; writing-original draft preparation, K.C.; writing-review and editing, K.K.; visualization, K.C.; supervision, K.K.; project administration, K.C. and K.K.; All authors have read and agreed to the published version of the manuscript.

Funding: This research received no external funding.

Institutional Review Board Statement: Not applicable.

Informed Consent Statement: Not applicable.

Data Availability Statement: Data are not available on a publicly accessible repository and they cannot be shared under request.

Acknowledgments: This paper was supported by the MSIT (Ministry of Science and ICT), Korea, under the ITRC (Information Technology Research Center) support program (IITP-2021-2018-0-01421) supervised by the IITP (Institute for Information \& communications Technology Promotion).

Conflicts of Interest: The authors declare no conflict of interest. 


\section{References}

1. Paul, T. Wide bandgap semiconductor power devices for energy efficient systems. In Proceedings of the 2015 IEEE 3rd Workshop on Wide Bandgap Power Devices and Applications (WiPDA), Blacksburg, VA, USA, 2-4 November 2015; pp. $402-405$.

2. Baliga, B.J. Fundamentals of Power Semiconductor Devices; Springer: New York, NY, USA, 2008.

3. Godignon, P.; Soler, V.; Cabello, M.; Montserrat, J.; Rebollo, J.; Knoll, L.; Bianda, E.; Mihaila, A. New Trends in High Voltage MOSFET Based on Wide Band Gap Materials. In Proceedings of the International Semiconductor Conference (CAS), Sinaia, Romania, 11-14 October 2017.

4. Zhang, M.; Wei, J.; Jiang, H.; Chen, K.J.; Cheng, C.H. Low ON-Resistance SiC Trench/Planar MOSFET With Reduced OFF-State Oxide Field and Low Gate Charges. IEEE Electron. Device Lett. 2016, 37, 1458-1461.

5. Cooper, J.A.; Agarwal, A. SiC power-switching devices-the second electronics revolution? Proc. IEEE 2002, 90, 956-968. [CrossRef]

6. Agarwal, A.; Baliga, B.J.; Francois, M.M.A.; Maxwell, E.; Berliner, N.; Papageorge, M. 3.3kV 4H-SiC Planar-Gate MOSFETs Manufactured using Gen-5 PRESiCE ${ }^{\mathrm{TM}}$ Technology in a 4-inch Wafer Commercial Foundry. In Proceedings of the SoutheastCon 2021, Atlanta, GA, USA, 10-13 March 2021; pp. 1-4.

7. Baba, M.; Tawara, T.; Morimoto, T.; Harada, S.; Takei, M.; Kimura, H. Ultra-Low Specific on-Resistance Achieved in 3.3 kV-Class $\mathrm{SiC}$ Superjunction MOSFET 2021. In Proceedings of the 3rd International Symposium on Power Semiconductor Devices and ICs (ISPSD), Nagoya, Japan, 30 May-3 June 2021; pp. 83-86.

8. Krishnaswami, S. Gate oxide reliability of $4 \mathrm{H}-\mathrm{SiC}$ MOS devices. In Proceedings of the IEEE International Reliability Physics Symposium (IRPS), San Jose, CA, USA, 17-21 April 2005; pp. 592-593.

9. Vudumula, P.; Kotamraju, S. Design and Optimization of 1.2-kV SiC Planar Inversion MOSFET Using Split Dummy Gate Concept for High-Frequency Applications. IEEE Electron. Device Lett. 2019, 66, 5266-5271. [CrossRef]

10. Han, K.; Baliga, B.J.; Sung, W. Split-Gate 1.2-kV 4H-SiC MOSFET: Analysis and Experimental Validation. IEEE Electron. Device Lett. 2017, 38, 1437-1440. [CrossRef]

11. Ishigaki, T.; Tatsunori, M.; Kinoshita, K.; Morikawa, T.; Oda, T.; Fujita, R.; Konishi, K.; Mori, Y.; Shima, A. 2019 Analysis of Degradation Phenomena in Bipolar Degradation Screening Process for SiC-MOSFETs. In Proceedings of the 31st International Symposium on Power Semiconductor Devices and ICs (ISPSD), Shanghai, China, 19-23 May 2019; pp. $259-262$.

12. Conrad, M.; De Doncker, R.W. Avoiding reverse recovery effects in super junction MOSFET based half-bridges. In Proceedings of the 2015 IEEE 6th International Symposium on Power Electronics for Distributed Generation Systems (PEDG), Aachen, Germany, 22-25 June 2015; pp. 1-5.

13. Kawahara, K.; Hino, S.; Sadamatsu, K.; Nakao, Y.; Yamashiro, Y.; Yamamoto, Y.; Iwamatsu, T.; Tomohisa, S.; Yamakawa, S. 6.5 kV schottky-barrier-diode-embedded SiC-MOSFET for compact full-unipolar module. In Proceedings of the 2017 29th International Symposium on Power Semiconductor Devices and IC's (ISPSD), Sapporo, Japan, 28 May-1 June 2017; pp. 41-44.

14. Soltau, N.; Wiesner, E.; Hatori, K. Switching Performance of 750A/3300V Dual SiC-Modules. Bodo's Power Syst. 2019, 2, 26-28.

15. Han, Z.; Bai, Y.; Chen, H.; Li, C.; Lu, J.; Song, G.; Liu, X. A trench/planar sic mosfet integrated with SBD (TPSBD) for low reverse recovery charge and low switching loss. Semicond. Sci. Technol. 2020, 35, 1-10. [CrossRef]

16. Synopsys, Inc. TCAD SentaurusTM Device User Guide; Synopsys, Inc.: Mountain View, CA, USA, 2017.

17. Sung, W.; Baliga, B.J. On Developing One-Chip Integration of $1.2 \mathrm{kV} \mathrm{SiC} \mathrm{MOSFET} \mathrm{and} \mathrm{JBS} \mathrm{Diode} \mathrm{(JBSFET).} \mathrm{IEEE} \mathrm{Trans.} \mathrm{Ind.}$ Electron. 2017, 64, 8206-8212. [CrossRef]

18. Pan, Y.; Tian, L.; Wu, H.; Li, Y.; Yang, F. 3.3kV 4H-SiC JBS diodes with single-zone JTE termination. Microelectron. Eng. 2017, 181, 10-15. [CrossRef]

19. Shenai, K.; Baliga, B.J. Monolithically integrated power MOSFET and Schottky diode with improved reverse recovery characteristics. IEEE Trans. Electron. Devices 1990, 37, 1167-1169. [CrossRef]

20. Ahn, J.; Ting, W.; Kwong, D.L. Comparison of performance and reliability between MOSFETs with LPCVD gate oxide and thermal gate oxide. IEEE Trans. Electron. Devices 1991, 38, 2709-2710. [CrossRef]

21. Saxena, R.S.; Kumar, M.J. Dual-Material-Gate Technique for Enhanced Transconductance and Breakdown Voltage of Trench Power MOSFETs. IEEE Trans. Electron. Devices 2009, 56, 517-522. [CrossRef]

22. Rao, V.R.; Eisele, I.; Patrikar, R.M.; Sharma, D.K.; Vasi, J.; Grabolla, T. High-field stressing of LPCVD gate oxides. IEEE Electron. Device Lett. 1997, 18, 84-86.

23. Sung, W.; Baliga, B.J. Monolithically Integrated 4H-SiC MOSFET and JBS Diode (JBSFET) Using a Single Ohmic/Schottky Process Scheme Applications. IEEE Electron. Device Lett. 2016, 37, 1605-1608. [CrossRef] 